\title{
Particle Theories. A Sketch of a Structuralist Reconstruction*
}

Teorías de partículas. Esbozo de una reconstrucción estructuralista

Joseph D. Sneed ${ }^{\dagger}$

\begin{abstract}
Particle theories intend to describe the fundamental constituents from which all matter is constructed and the interactions among them. These constituents include atoms and molecules as well as their subatomic constituents, nuclei and their component parts including elementary particles. We consider an alternative to the usual particle theories (PT's), but dealing with the same phenomena. We call these theories 'QT's'. This is an attempt to provide a formal description of the essential features of elementary particle theories within the framework of metatheoretical structuralism.
\end{abstract}

Keywords: particle - theories - rational reconstruction - metatheoretical structuralism

\section{Resumen}

Las teorías de partículas intentan describir los constituyentes fundamentales a partir de los cuales se construye toda la materia y las interacciones entre éstos. Estos constituyentes incluyen átomos y moléculas, así como sus constituyentes subatómicos, núcleos y sus partes componentes, incluyendo partículas elementales. Consideramos una alternativa a las teorías de partículas (PT) usuales pero que tratan sobre los mismos fenómenos. Llamamos a estas teorías 'QT's'. Este es un intento de proporcionar una descripción formal de los rasgos esenciales de las teorías de partículas en el formato del estructuralismo metateórico.

Palabras clave: partículas - teorías - reconstrucción racional - estructuralismo metateórico

\footnotetext{
* Received: 5 October 2019. Accepted: 7 January 2020.

Editors' Note: This is a posthumous paper by Joseph D. Sneed (1938-2020). The story of this paper is as follows. Some time ago Joseph D. Sneed ("Joe") distributed among colleagues reports of his recent work in progress on elementary particle theories in an e-mail reminding us of his advanced age and telling us that he was not in good health. He continued to work on those reports, which, some time later, he shaped into an article and submitted to Metatheoria. Regrettably, the time spent during the peer review process and in preparation for publication prevented Sneed from seeing his paper published. We paid tribute to him with the posthumous publication of his article.

+ Colorado School of Mines, USA.

Metatheoria11(1)(2020): 33-52. ISSN 1853-2322. eISSN 1853-2330.

(C) Editorial de la Universidad Nacional de Tres de Febrero.

(C) Editorial de la Universidad Nacional de Quilmes.

Publicado en la República Argentina.
} 


\section{Introduction}

Particle theories pretend to describe the fundamental constituents from which all matter is constructed and the interactions among them. These constituents include atoms and molecules as well as their subatomic constituents, nuclei and their component parts including elementary particles.

The set of particle theories is

$$
\mathrm{PT}=\{\mathrm{X} / \mathrm{X} \text { is a particle theory }\} \text {. }
$$

Informally, we refer to a member of PT as 'a PT' and PT as 'PT's'. We consider an alternative to the usual particle theories (PT's), but dealing with the same phenomena.

We call these theories 'QT's'; a single such theory is called 'a QT'.

This is essentially a structuralist reconstruction of particle theories, (2.2) below (Balzer, Moulines \& Sneed 1987).

\section{Tracks}

The data for QT is tracks, things that are observed in things like cloud/bubble chambers. The objective of the theory is to characterize all and only observed tracks, i.e. describe a set that contains all and only observed tracks.

We assume we have descriptions of all tracks ever observed (lots, $\sim 10^{6}$ ). From this data we invent particles and interactions that characterize all and only observed tracks.

That "particles" and "elementary particles" appear in this characterization is accidental. We do not set out to look for them.

This "turns history on its head". Nothing at all like this happened. But looking at things in this way provides insight into the way the usual PT's work.

The general configuration of tracks is this. Tracks have diverging points where one trajectory stops and several others begin as well as converging points where several tracks converge to one track.

The basic data for a QT is provided by detectors.

Most detection mechanisms rely on the fact that when high-energy charged particles pass through matter they ionize atoms along their path [...] electrically neutral particles do not cause ionization [...] their paths have been reconstructed by analyzing the tracks of the charged particles [...] invoking conservation of energy and momentum at each vertex (Feynman, Leighton \& Sands 1965, p.8).

We understand this as "most, but not all". Relentlessly carrying out this view requires a somewhat more general conception of "detector".

Here we try to describe QT's with a minimum of metaphorical interpretation. Detector data is described as tracks. A track is an n-indexing of the set

$$
\operatorname{Tr}=\left\{\gamma \in \operatorname{SET}\left(T, \mathbb{R}^{2}\right) \mid \mathrm{T} \in \operatorname{INT}\left(\mathbb{R}^{1}\right)\right\},
$$

where $\gamma$ is a curve in $\mathbb{R}^{2}$ so that $\gamma(t)$ is a 2 -tuple of real numbers.

$\mathrm{T}$ is an interval of real numbers interpreted as "time". One might take T to be the same in all QT's or let $\mathrm{T}$ be specific to each QT. It does not appear to matter. To make everything explicit, we choose the second option. Thus, notation like

$$
\text { ' } \mathrm{T}_{\mathrm{QT}} \text { ' }
$$

is meaningful, though we do not use it.

We let

$$
\stackrel{\mathrm{n}}{\operatorname{Tr}}=\text { n-index } \in \operatorname{SET}\left(\left\{\gamma \in \operatorname{SET}\left(T, \mathbb{R}^{2}\right) \mid \mathrm{T} \in \operatorname{INT}\left(\mathbb{R}^{1}\right)\right\}, \mathbb{I}^{[1, \mathrm{n}]}\right)
$$




$$
\begin{aligned}
\operatorname{Tr}= & \text { n-index } \in \operatorname{SET}\left(T-P A T H-\mathbb{R}^{2}, \mathbb{I}^{[1, n]}\right) \\
& \operatorname{Tr}_{i} \text { is the value of } \operatorname{Tr}^{n} \text { at } i .
\end{aligned}
$$

Thus, the set of all $\mathbf{n}$-tracks is

$$
\{\text { n-index } \mid \mathrm{n} \in \mathbb{I}\}=\{\stackrel{\mathrm{n}}{\operatorname{Tr}} \mid \mathrm{n} \in \mathbb{I}\} .
$$

A vertex is located at $\mathrm{t}^{\prime} \in \mathrm{T}$ just when

$$
\operatorname{Tr}_{1}\left(t^{\prime}\right)=\operatorname{Tr}_{2}\left(t^{\prime}\right)=\ldots=\operatorname{Tr}_{n}\left(t^{\prime}\right)
$$

where

$$
\mathrm{t}<\mathrm{t}^{\prime} \sim \text { before; } \mathrm{t}>\mathrm{t}^{\prime} \sim \text { after. }
$$

There may be multiple vertices on the same track.

Generally, tracks are 3-dimensional in that $\gamma \in \operatorname{SET}\left(T, \mathbb{R}^{3}\right)$ and $\gamma(\mathrm{t}) \in \mathbb{R}^{3}$. Cloud chamber photographs, etc., are 2-dimensional. But, via an intricate argument, they are viewed as cross- sections of 3-dimensional paths (Kane 2016, p. 9). Note that tracks are most generally continuous curves in $\mathbb{R}^{3}$ which may include straight line segments.

Tracks are "interpretations" of more basic data. The photograph appearing in Feynman, Leighton and Sands (1965, p. 46) is a configuration of ink dots on the page in my copy of the book. It is "interpreted" as set of tracks.

This configuration of dots is derived by the halftone (or much less likely photogravure) process from a gelatin silver print which consists of small particles of silver bound in a layer of gelatin.

The basic empirical data is usually a gelatin silver print. The process of converting this data to what I see on the book page is an example of the "interpretation" described in general in (Kane 2016, p. 9).

Ultimately this is a manifestation of the holistic character of empirical theories described in Balzer, Moulines and Sneed (1987).

Roughly, tracks are basic data relative to particle theories (QT's). Treating them in this way permits us to provide a systematic description of these theories.

\section{Particle tracks}

In the beginning there were tracks. With trial and error, man gave us particles and assigned them to particle tracks until the given tracks were reproduced. This was recorded for history by Gordy Kane (Kane 1958).

First, we define a particle zoo (2.1), then n-particle tracks. Intuitively, an n-particle track is a function whose range is a set of $\mathrm{n}$-tracks and whose domain is a particle zoo; i.e. a member of

$$
\operatorname{SET}(\{\text { n-index } \mid \mathrm{n} \in \mathbb{I}\}=\{\stackrel{\mathrm{n}}{\mathrm{T}} \mathrm{r} \mid \mathrm{n} \in \mathbb{I}\} \text {, particle zoo). }
$$

\subsection{Particle zoo}

A particle zoo is a finite set of particle types. A particle type is a set of 〈property, values〉 pairs where property is an individual and values is a set. Thus, $\left\langle\right.$ mass, $\left.\mathbb{R}^{+}\right\rangle$is a property type. Intuitively, property is the name of the property and values is the set of values the property may have.

Particles are assumed to satisfy the laws of particle mechanics and electrodynamics, most generally relativistic but, classical in limiting cases. This assumption entails that particles are small, spatially confined objects, satisfying the conservation laws for these theories. In the structuralist terminology, they are parts of models for these theories. Thus, all particle types have mechanical properties like 
$\left\langle\right.$ position, $\left.\mathbb{R}^{3}\right\rangle$, $\left\langle\right.$ velocity, $\left.\mathbb{R}^{3}\right\rangle$. Usually, we will specify only additional, non-mechanical, properties in describing particle types below (4).

It is this assumption that leads to the "forces" in QT's. If a particle consists of 2 particles with charge (e.g. protons), there must be a "force" (e.g. the "strong force") holding it together.

It requires that all particles are subject to some forces, though not necessarily a familiar one. This is why we assume the existence of a "weak force" mediated by mesons.

Conservation laws for these properties restrict the set of possible particle tracks. Roughly, conservation laws require that the sums of the property values before and after the collision are identical.

Some properties may be defined in terms of others, e. g. kinetic energy in terms of mass and velocity.

A set of particle properties is a complete set iff all particle properties can be defined in terms of members of the set. A complete set is minimal if no subset of it is complete.

The state of a particle is a specification the values of a minimal complete set of properties of the particle.

The state of a particle changes over time in a way that is described by particle mechanics.

The full apparatus of particle mechanics can be applied to these particles, including defined properties like energy, linear and angular momentum. Lagrangian and Hamiltonian formulations of these theories may be employed.

A single particle zoo is associated with each QT at any point in time so that the notation

$$
\mathrm{ZOO}_{\mathrm{QT}, \mathrm{t}}
$$

is well defined. Nuclear particles and elementary particles are subsets of $\mathrm{ZOO}_{\mathrm{QT}, \mathrm{t}}$ so that

$$
\mathrm{NZOO}_{\mathrm{QT}, \mathrm{t}} \subseteq \mathrm{ZOO}_{\mathrm{QT}, \mathrm{t}} \quad \mathrm{EZOO}_{\mathrm{QT}, \mathrm{t}} \subseteq \mathrm{ZOO}_{\mathrm{QT}, \mathrm{t}}
$$

though it is not the case that

$$
\mathrm{EZOO}_{\mathrm{QT}, \mathrm{t}} \subseteq \mathrm{NZOO}_{\mathrm{QT}, \mathrm{t}}
$$

Elementary particles are not nuclear particles.

Particles come to be in the zoo when they are found to reproduce specific observed track data (e.g. segments of circles, convergence and divergence). Their properties and specific values of these properties are found which, when combined with specific laws (e.g. the cyclotron radius equation), reproduce the observed track data. This is just structuralist determination of values of theoretical functions (Balzer, Moulines \& Sneed 1987). The zoo is populated in this way. Particles are never removed from the zoo...unless the observed track data on which they are based is discovered to be in error. The zoo is found to be in need of enlargement when track data is observed which cannot be reproduced in this way by particles currently in the zoo.

\subsection{Description}

We consider n-particle tracks. The set of 1-particle tracks is

$$
\operatorname{PTr}=\operatorname{SET}\left(\operatorname{Tr}^{1}\right. \text {, particle zoo). }
$$

Where particle zoo is a set of particle types described above (2.1) and

$$
\mathrm{a}(\mathrm{Tr}) \sim \text { particle type assigned to the track } \mathrm{Tr} .
$$

Thus, the set of n-particle tracks is

$$
\{\text { n-index } \mid \mathrm{n} \in \mathbb{I}\}=\{\stackrel{\mathrm{n}}{\operatorname{Pr}} \mid \mathrm{n} \in \mathbb{I}\} .
$$

The set of particle tracks is 


$$
\left\{\text { n-index } \mid \mathrm{n} \in_{\odot}\right\}=\{\stackrel{\mathrm{n}}{\mathrm{Pr}} \mid \mathrm{n} \in \mathbb{I}\} \text {. }
$$

A vertex on a particle track is located at $t^{\prime} \in \mathrm{T}$ just when

$$
\operatorname{Pr}_{1}\left(t^{\prime}\right)=\operatorname{Pr}_{2}\left(t^{\prime}\right)=\ldots=\operatorname{Pr}_{n}\left(t^{\prime}\right)
$$

where

$$
\mathrm{t}<\mathrm{t}^{\prime} \sim \text { before; } \mathrm{t}>\mathrm{t}^{\prime} \sim \text { after. }
$$

Note that ' $\operatorname{Pr}_{\mathrm{n}}(\mathrm{t})$ ' denotes a single number associated with particle $\mathrm{n}$. If there are two numbers associated with time $t$, these numbers must be associated with different particles. Intuitively, particle tracks cannot "overlap".

There are observed particle tracks on which there are vertices where the track:

1) simply changes direction;

2) diverges indicating the appearance (creation, birth) of particles composed of parts of the initial particle;

3) converges indicating the appearance of a particle composed of the converging particles (annihilation, decay).

We call all these (1, 2, and 3 above) ' $\Delta$ P-events'.

Divergence ( 2 , above) may be viewed as the particle's fragmenting into its constituent parts. Convergence (3, above) may be viewed as the particle's constituent parts uniting to form the particle.

A particle is elementary (3.4) iff all observed particle tracks in which the particle appears terminate without diverging (2, above), i.e. it is has never been observed to fragment into constituent parts.

The set of elementary particles observed at time $t$ is

$$
\mathrm{EZOO}_{\mathrm{QT}, \mathrm{t}} \subseteq \mathrm{ZOO}_{\mathrm{QT}, \mathrm{t}} .
$$

As indicated, it is a subset of $\mathrm{ZOO}_{\mathrm{QT}, \mathrm{t} .}$. Intuitively, it is a proper subset. But, it is at least logically possible that all particles in the $\mathrm{ZOO}_{\mathrm{QT}, \mathrm{t}}$ are elementary. Observed tracks never diverge and never converge.

The photograph appearing in Feynman, Leighton and Sands $(1965$, p.46) is a set of tracks. The diagram appearing on the same page is a set of particle theories. The labels on the diagram are values of the assignment function a.

Particle tracks may be described in terms pairs of functions,

$$
\langle\text { cross section function, decay rate function }\rangle=\langle\sigma, \Gamma\rangle
$$

$$
\sigma \in \operatorname{SET}\left(T, \mathbb{R}^{3}\right), T \in \operatorname{SET}\left(T, \mathbb{R}^{1}\right) .
$$

Intuitively, $\sigma(t)$ is the curvature of the path at time $t$. It is a 3 -vector whose components are the curvature of the path at time $t$ in each direction. $\Gamma(t)$ is the speed of the particle along the curve at time t.

The differential geometry of describing particle tracks in this way is non-trivial. This is not considered here.

Let

$$
\sigma \mathrm{T}=\left\{\left\langle\sigma \in \operatorname{SET}\left(\mathrm{T}, \mathbb{R}^{3}\right), \mathrm{T} \in \operatorname{SET}\left(\mathrm{T}, \mathbb{R}^{1}\right)\right\rangle \mid \mathrm{T} \in \mathrm{INT}(\mathbb{I})\right\} .
$$

$\sigma \mathrm{T}$ is the set of all $\langle$ cross section function, decay rate function $\rangle$ pairs.

$\langle$ cross section function, decay rate function〉 pairs correspond to particle tracks via the function

$$
\begin{gathered}
\chi \in \operatorname{SET}(\sigma \mathrm{T},\{\mathrm{n} \operatorname{Pr} \mid \mathrm{n} \in \mathbb{I}\}) \text { such that } \\
\chi(\sigma \mathrm{T})=\langle\sigma, \operatorname{Pr} \mid \mathrm{n} \in \mathbb{I}\rangle \Leftrightarrow \forall \mathrm{i} \in \operatorname{INT}[1, \mathrm{n}], \mathrm{P}_{\mathrm{i}}=\mathrm{T}
\end{gathered}
$$


T determines the length of the particle path. It is paired with anycross section function in the values of the $\chi$-function.

In the structuralist jargon

$$
\{\text {-index } \mid \mathrm{n} \in \mathbb{I}\}=\{\stackrel{\mathrm{n}}{\operatorname{Pr}} \mid \mathrm{n} \in \mathbb{I}\}=\mathbf{M}_{\mathrm{pp}}[\mathrm{QT}] \text {. }
$$

Theoretical structures are obtained by assigning mechanical properties to particles. Roughly, particles are theoretical concepts in QT.

The particle zoo is essentially $\mathbf{M}_{\mathrm{p}}[\mathrm{QT}]$.

We choose a particle in the zoo and see if the tracks it produces reproduce the non-theoretical tracks. If they do, we win. If they do not, we try another particle. If no member of the zoo works, we look to expand the zoo, i.e. try to find a new particle.

\subsection{Probabilities}

$\Delta \mathrm{m} \Delta \mathrm{P}$-events are ordered, i.e. appear in a partial ordering

$$
\mathrm{p}\langle\Delta \mathrm{m} \Delta \mathrm{P} \text {-events, } \preccurlyeq\rangle \in \mathrm{PART} \text { ORD }
$$

We want to describe how prior $\Delta \mathrm{m} \Delta \mathrm{P}$-events influence subsequent $\Delta \mathrm{m} \Delta \mathrm{P}$-events. To do this we consider a probability function $\mathrm{p}$ on $\Delta \mathrm{m} \Delta \mathrm{P}$-events.

$$
\mathrm{p} \in \operatorname{PROB}(\Delta \mathrm{m} \Delta \mathrm{P} \text {-events }) .
$$

For

$$
\Delta \mathrm{m} \Delta \mathrm{P} \text {-events } \preccurlyeq \Delta \mathrm{m} \Delta \mathrm{P}_{\text {-events' }}
$$

we are interested in

$$
\mathrm{p}(\Delta \mathrm{m} \text { P-events } \mid \Delta \mathrm{m} \text { P-events'). }
$$

These probabilities correspond to different || -embeddings.

We are interested in conditional probabilities:

$$
\mathrm{p}(\text { P-event }) \mid \text { P-event'). }
$$

These conditional probabilities are determined by QM in the following way. Consider a probability function on $\sigma \mathrm{T}$

$$
\mathrm{p} \in \operatorname{prob}(\sigma \Gamma) .
$$

The details of the calculation involve Feynman Diagrams.

[...] we begin the quantitative formulation of elementary particle dynamics which amounts, in practice, to the calculation of decay rates $(\Gamma)$ and scattering cross sections $(\sigma)$. The procedure involves two distinct parts: (1) evaluation of the relevant Feynman diagram for the process in question and (2) to determine the 'amplitude' (M) for the process in question and (2) insertion of M into Fermi's "Golden Rule" to compute $\Gamma$ or $\sigma$ as the case may be (Griffiths 2000, p. 64).

Each vertex is a $\sigma \Gamma$ and corresponds to a particle track via the $\chi$-function.

Two (or more) vertices on the same particle track can be seen as a superposition of two QM states. This is the way to treat "neutrino oscillations" and "resonances".

Most generally, an m-tuple of vertices on the same particle track is located at $\mathrm{t}^{\prime} \in \mathrm{T}$ just when

$$
\begin{gathered}
\mathrm{m}_{1}(\mathrm{t}) \operatorname{Pr}_{1}\left(\mathrm{t}^{\prime}\right)=\mathrm{m}_{2}(\mathrm{t}) \operatorname{Pr}_{2}\left(\mathrm{t}^{\prime}\right)=\ldots=\mathrm{m}_{\mathrm{m}}(\mathrm{t}) \operatorname{Pr}_{\mathrm{n}}\left(\mathrm{t}^{\prime}\right) \\
\mathrm{t}<\mathrm{t}^{\prime} \sim \text { before; } \mathrm{t}>\mathrm{t}^{\prime} \sim \text { after }
\end{gathered}
$$

m-tuples of vertices on the same particle track indicate the appearance (creation), disappearance (annihilation) and trajectory change of $\mathrm{m}$ particles. We call these ' $\Delta \mathrm{m} \Delta \mathrm{P}$-events'. 
Each of these || -embeddings corresponds to a weighted sum of $\mathrm{m} \Delta \mathrm{P}$-embeddings. This is a consequence of a general property of QM-states, the superposition principle.

\subsection{Elementary particles}

Observed elementary particles are commonly classified according to a scheme like that exhibited below.

\begin{tabular}{|c|c|c|c|}
\hline & ELEMENTARY PARTICLES & & \\
\hline \multicolumn{2}{|l|}{ FERMIONS } & \multicolumn{2}{|l|}{ BOSONS } \\
\hline \multicolumn{2}{|l|}{ Spin $1 / 2$ integer } & \multicolumn{2}{|l|}{ Spin 1 integer } \\
\hline \multicolumn{2}{|l|}{ Pauli exclusion } & \multicolumn{2}{|l|}{ Bose-Einstein } \\
\hline QUARKS/ANTI QUARKS & LEPTONS/ANTI LEPTONS & GAUGE & SCALAR \\
\hline Color charge, strong interaction & No color charge, weak interaction & $\begin{array}{c}\text { Spin } \neq 0, \text { force } \\
\text { carriers }\end{array}$ & Spin $=0$ \\
\hline Generations & Generations & Four kinds & Higgs \\
\hline 1. Up $(\mathrm{u})$, Down $(\mathrm{d})$ & $\begin{array}{l}\text { 1. Electron }\left(\mathrm{e}^{-}\right) \text {, Electron neutrino } \\
\qquad\left(\mathrm{v}^{-}\right)\end{array}$ & 1. Photons $(\gamma)$ & \\
\hline 2. Charm (c), Strange (s) & 2. Muon $\left(\mu^{-}\right)$, Muon neutrino $\left(v_{\mu}\right)$ & 2. W Z bosons & \\
\hline \multirow[t]{2}{*}{ 3. Top (t), Bottom (b) } & 3. Tau $\left(\tau^{-}\right)$, Tau neutrino $\left(v_{\tau}\right)$ & 3.8 types gluons & \\
\hline & & 4. Graviton $(\mathrm{G})$ & \\
\hline
\end{tabular}

\section{QM}

Most generally, QM tells us how earlier and later features (divergence and convergence) of particle tracks are related. It does this using a probability function defined on $\sigma \Gamma$.

In the application of QM here, the || -embedding (3.8) is constrained by additional conditions which effectively determine the probabilities. These conditions are described by Feynman diagrams and related calculations. These will not be considered in detail here. For our purposes it suffices to know the role they play.

Material in this section has wider application than particle tracks. In an ideal world, it would be part of a "tool kit" to be employed as needed in a variety of contexts. In this real world it must be expounded anew whenever it is used.

The view taken here is that QM is not a part of elementary particle physics; it is not a physical theory at all. QM is most appropriately viewed as a branch of mathematical probability theory. An earlier formulation of this view of QM is appears in Sneed (2011).

\subsection{Systems}

Members of $\sigma \Gamma$ are viewed as "systems". Systems are sets of "states". Any Boolean algebra can be "quantized" in this way. The essential step is the ||-EMBEDDING (3.8). Intuitively, members $\sigma \Gamma$ of are viewed as "systems", non-QM states; their ||-images are QM-states.

$\mathrm{X}$ is the set of elementary states of system $\mathrm{X}$. Essentially, systems are sets of elementary states. POT $\mathrm{X}$ is the set of states of system $\mathbf{X}$. $\mathbb{S}$ is the set of all systems.

There is a concatenation operation on $\mathbb{S}$. The concatenation of systems $\mathbf{X}$ and $\mathbf{Y}$ is denoted by ' $\mathrm{XY}$ '. $\mathrm{XY}$ is a proper subset of $\mathrm{X} \times \mathrm{Y}$, i.e.

$$
\mathrm{XY} \subset \mathrm{X} \times \mathrm{Y} .
$$

Members of $\mathrm{X} \times \mathrm{Y}$ are product states. Members of $\mathrm{XY}-\mathrm{X} \times \mathrm{Y}$ are non-product states.

In more detail, and more systematically (at the price of some redundancy) systems live in a system structure 


$$
\mathbb{S}=\left\langle\mathbb{S}, \approx, \mathrm{l},{ }^{\circ}\right\rangle
$$

where $\mathbb{S}$ is a finite set.

Members of $\mathbb{S}$ are systems, finite sets

$$
\mathrm{X} \in \mathbb{S} \subset|\mathrm{SET}|,
$$

$\approx$ is an equivalence relation on $\mathbb{S}$ and $\mathbf{l}$ an isomorphism function

$$
\imath \in \operatorname{SET}(\approx,|| \operatorname{SET}||)
$$

such that, for all $\langle\mathrm{X}, \mathrm{Y}\rangle \in \approx$,

$$
\iota_{\mathrm{X}}^{\mathrm{Y}}:={ }_{\mathfrak{l}}(\mathrm{X}, \mathrm{Y}) \in \operatorname{ISET}(\mathrm{X}, \mathrm{Y}) .
$$

Intuitively, $\approx$-equivalent systems are systems of the same kind, e.g. electrons.

The mere existence of $\mathbf{l}$ entails only that equivalent systems have the same cardinality. However, we take $\boldsymbol{l}$ to be a specific isomorphism which is determined by unspecified internal properties of the states. For example, the states of electrons are isomorphic in some electron-specific way.

${ }^{\circ}$ is a binary operation on $\mathbb{S}$

$$
{ }^{\circ} \in \operatorname{SET}(\mathbb{S} \times \mathbb{S}, \mathbb{S})
$$

such that

$$
\mathrm{X}^{\circ} \mathrm{Y} \subset \mathrm{X} \times \mathrm{Y} .
$$

In the context of $\mathrm{QM},{ }^{\circ}$ is called 'concatenation' and

$$
\mathrm{XY}:=\mathrm{X}^{\circ} \mathrm{Y} .
$$

Members of $\mathrm{X} \times \mathrm{Y}$ are product states. Members of $\mathrm{XY}-\mathrm{X} \times \mathrm{Y}$ are non-product states.

Equivalence and isomorphism are required to be "consistent" with concatenation

$$
\begin{gathered}
\iota_{\mathrm{X}}^{\mathrm{Y}} \iota_{\mathrm{Z}}^{\mathrm{W}} \Rightarrow \iota_{\mathrm{XZ}}^{\mathrm{YW}} \\
\mathrm{X} \approx \mathrm{Y}, \mathrm{Z} \approx \mathrm{W} \Rightarrow \mathrm{XY} \approx \mathrm{ZW} .
\end{gathered}
$$

Concatenation of distinct systems of the same kind, e. g. two electrons, is intuitively unproblematic. How to understand concatenation of identical systems is less clear. Since it appears to be formally unproblematic, we do not rule it out.

We require that $\approx$-equivalence is such that the order of concatenation is irrelevant, so we require

$$
\mathrm{XY} \approx \mathrm{YX} .
$$

For most purposes, compound systems like $\mathrm{XY}$ are treated like generic systems. The internal structure of states of generic systems is not specified. The internal structure of the states of compound systems is made more explicit in that they are ordered pairs and, more generally, ordered tuples.

It is possible to consider a set of elementary systems

$$
\mathbb{E} \subseteq \mathbb{S}
$$

such that all systems in $\mathbb{S}$ are ${ }^{\circ}$-concatenations systems in $\mathbb{E}$. System structure would then be

$$
\mathfrak{S}=\langle\mathbb{S}, \approx, \mathrm{l}, \stackrel{\circ}{ }, \mathbb{E}\rangle .
$$

However, this is not essential to QM. The set

$$
\$:=\bigcup_{\mathrm{X} \in \mathrm{S}} \operatorname{Pot} \mathbf{X}
$$

is the set of all states of all systems. Members of each member of $\$$ all belong to the same system. Since systems do not intersect, members of $\$$ effectively assign a state to each system. 
Some members of $\mathbb{S}$ are sets of singletons, essentially sets of elementary systems, these are effectively an elementary state assignments to each system. Because the probabilities of interest are defined on states rather than elementary states we need to assign a state to each system.

\subsection{Boolean algebra}

The Boolean algebra of sub-sets of $\mathbf{T}$-sequences of members of $\$$ is

$\mathrm{B}_{\$ \times \mathrm{T}}$.

This is the Boolean algebra on which the probability functions relevant to QM are defined.

\subsection{Observables}

Partitions of system $\mathbf{X}$ are non-null sets of mutually exclusive, jointly exhaustive states. Certain partitions are observables. For observable partitions, the member of the partition containing the state of the system can be determined. We do not explain how this might be done. Roughly, it is done by the system's interacting with some other system in the manner described in (3.6-3.7) below.

For system $\mathbf{X}$, the partition lattice is

$$
\mathrm{V}_{\mathrm{X}}=\left\langle\left|\mathrm{V}_{\mathbf{X}}\right|, \leq_{\mathbf{X}}^{\mathrm{V}}, \mathrm{V}_{\mathbf{X}}^{\mathrm{V}}, \wedge_{\mathbf{X}}^{\mathrm{V}}, \overline{\mathrm{V}}_{\mathbf{X}}^{\mathbf{V}}, \bar{\Lambda}_{\mathbf{X}}^{\mathbf{V}}\right\rangle .
$$

Partitions and their members are indexed so that $o_{\mathbf{X}}^{\mathbf{i}} \in\left|V_{\mathbf{X}}\right|$ is an indexed partition of $\mathbf{X}$, and ${ }^{i} o_{\mathbf{X}}^{\mathbf{i}} \in o_{\mathbf{X}}^{\mathbf{i}}$ is an indexed state in partition $o_{\mathbf{X}}^{\mathbf{i}}$.

For compound product systems:

$$
{ }^{k} O_{\mathbf{X Y}}^{\mathbf{k}}={ }^{k} O_{\mathbf{i}}^{\mathbf{X}} \times \mathbf{Y} \in v_{\mathbf{X Y}}^{\mathbf{k}}
$$

For non-product systems:

$$
{ }^{k} o_{\mathrm{XY}}^{\mathrm{k}} \in v_{\mathrm{XY}}^{\mathrm{k}}
$$

For observable partitions, the member of the partition containing the state of the system can be determined. We do not explain how this might be done. Roughly, it is done by the system's interacting with some other system in the manner described in (3.6-3.7) below.

For $v_{\mathrm{x}} \in\left|\mathrm{V}_{\mathrm{x}}\right|, \uparrow_{\mathrm{v}_{\mathrm{x}}}$ is the set of all partitions coarser (less fine) than $v_{\mathrm{x}}$. The set of observables for system $\mathbf{X}$ is

$$
\Omega_{\mathrm{X}} \subseteq\left|\mathrm{V}_{\mathrm{X}}\right|
$$

We require
1) $\Omega_{\mathrm{X}} \neq \Lambda$
2) $v_{x} \in \Omega_{x} \Rightarrow \uparrow_{\mathrm{x}} \subset \Omega_{\mathrm{x}}$
3) $v_{\mathrm{X}} \in \Omega_{\mathrm{X}} \Rightarrow v_{\mathrm{X}} \times \mathrm{Y} \in \Omega_{\mathrm{XY}}$.

All systems have at least one observable 1); there are no unobservable systems; if $v_{x}$ is observable all partitions coarser than $v_{x}$ are observable 2); if the member of the partition $v_{x}$ containing the state of the system can be determined then the member containing the state of all partitions coarser than $v_{X}$ can be determined. To see this note that, for $v^{\prime} x \in \uparrow v_{x}$, every member of $v^{\prime} x$ contains exactly one member of $v_{\mathbf{X}}$, say ${ }^{i} o_{\mathbf{X}}^{\mathbf{i}}$. Thus, if ${ }^{i} o_{\mathbf{X}}^{\mathbf{i}}$ is determined to contain the state of $\mathbf{X}$ then ${ }^{i} o_{\mathbf{X}}^{\mathbf{i}^{\prime}}$ is determined to contain the state of $\mathbf{X}$; if $v_{\mathbf{X}}$ is an observable for $\mathbf{X}, v_{\mathbf{X}} \times \mathbf{Y}$ is an observable for $\mathbf{X Y} 3$ ); if ${ }^{i} o_{\mathbf{X}}^{\mathbf{i}}$ is determined to contain the state of $\mathbf{X}$ then ${ }^{i} o_{\mathbf{X}}^{\mathbf{i}} \times \mathbf{Y}$ is determined to contain the state of $\mathbf{X Y}$.

The partition ordering, restricted to $\Omega_{\mathrm{X}}$, is a sub-lattice of $\mathrm{V}_{\mathrm{X}}$,

$$
\mathrm{L}_{\mathrm{X}}^{\Omega} \sqsubset_{\mathrm{LAT}} \mathrm{V}_{\mathrm{x}} \text {. }
$$

Atoms of $\mathbf{L}_{\mathbf{X}}^{\Omega}$ are $\widehat{v}_{\mathbf{X}}^{\mathbf{i}}$. 


\subsection{Probabilities}

QM is a theory about certain conditional probabilities derived from probability functions

$$
\mathrm{p} \in \operatorname{PROB}\left(\mathrm{B}_{\$ \times \mathrm{T}}\right)
$$

where $\operatorname{PROB}(\mathrm{B})$ is the set of all probability functions over the Boolean algebra $\mathbf{B}$. The formal properties of these functions are described in modern treatments of mathematical probability theory.

We assume that statements about values of $\mathbf{p}$ for all members of $\left|\mathbf{B}_{\mathrm{T}}\right|$ are "meaningful" in the sense that their truth conditions are well understood. That these probabilities actually have the values determined by QM is an empirical claim. Intuitively, these are probabilities of individual events which are determined, in some way, by facts about these individual events. However, QM has nothing to say about individual events nor the way in which facts about them determine probabilities.

More generally, QM is not committed to any specific view about the appropriate "interpretation" (e.g. personalistic, relative frequency, ensemble, propensity interpretations) of probability function values. There is a large body of quantum mechanical literature pertaining to this question. We do not offer a contribution to this literature. However, we do note that the personalistic interpretation, long defended by Jaynes (2003) as appropriate to statistical mechanical probabilities, has recently been suggested to be uniquely appropriate to quantum mechanics (Caves, Fuchs \& Schack 2002). Further, the Boolean algebra providing the domain for the probability functions used in QM is so large and loosely specified that anything like a frequency interpretation seems somewhat implausible.

Most generally, we consider, for $\mathrm{o} \in \boldsymbol{\$}$,

$$
\mathrm{p}(\mathrm{o}, \mathrm{t}):=\mathrm{p}(\langle\mathrm{o}, \mathrm{t}\rangle)
$$

Note that

$$
{ }^{i} o_{\mathbf{X}}^{\mathbf{i}} \in \$
$$

so that

$$
\mathbf{p}\left({ }^{i} o_{\mathbf{X}}^{\mathbf{i}}, t\right)
$$

is defined.

\subsection{Conditional probabilities}

QM is concerned with conditional probabilities of the form

$$
\left.\mathbf{p}\left({ }^{k} o_{\mathbf{X}}^{\mathbf{i}}, t\right) \mid{ }^{(i j} o_{\mathbf{X Y}}^{\mathbf{I J}}, \mathrm{t}-\mathrm{n}\right),
$$

the probability that component system $\mathrm{X}$ is in state ${ }^{k} o_{\mathbf{X}}^{\mathbf{i}}$ at time $t$, given that the compound system $\mathrm{XY}$ is in state ${ }^{i j} o_{\mathbf{X Y}}^{\mathbf{I J}}$ at $\mathrm{t}-\mathrm{n}$. These might be called 'partial transition probabilities'.

${ }^{i j} O_{\mathbf{X Y}}^{\mathbf{I J}}$ may be interpreted as the initial state of an interaction that occurred over the time interval [t $-\mathrm{n}, \mathrm{t}] .{ }^{k l}{ }^{\mathrm{IJ}} \mathbf{X Y}$ is the final state.

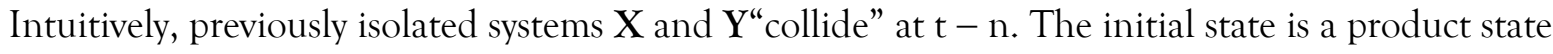

$$
\left.\mathbf{p}{ }^{(i j} o_{\mathbf{X Y}}^{\mathbf{I J}}, \mathrm{t}-\mathrm{n}\right)=\mathbf{p}\left({ }^{\mathrm{i}} o_{\mathbf{X}}^{\mathbf{I}}, \mathrm{t}-\mathrm{n}\right) \mathbf{p}\left({ }^{(j} o_{\mathbf{Y}}^{\mathbf{J}}, \mathrm{t}-\mathrm{n}\right)
$$

In the simplest case, the "final state" is also a product state so that

$$
\mathbf{p}\left({ }^{k l} O_{\mathbf{X Y}}^{\mathbf{I J}}, \mathrm{t}-\mathrm{n}\right)=\mathbf{p}\left({ }^{k} o_{\mathbf{X}}^{\mathbf{I}}, \mathrm{t}-\mathrm{n}\right) \mathbf{p}\left({ }^{\mathrm{l}} o_{\mathbf{Y}}^{\mathbf{J}}, \mathrm{t}-\mathrm{n}\right) .
$$

It is not obvious that the final state should be viewed as a product state. The Stern-Gerlach example suggests otherwise. Taking it to be a product state is simple, but not essential to the formal development when the || -embedding operates on the compound system XY.

These probabilities, for all pairs of systems, together with $\Omega_{\mathbf{X}}$, are $\mathbf{M}_{\mathrm{pp}}$,

$$
\mathbf{M}_{\mathrm{pp}}=\left\langle\Omega_{\mathbf{X Y}}, \mathbf{p}^{(k l} o_{\mathbf{X Y}}^{\mathbf{I J}}, \mathrm{t}-\mathrm{n}\right)\left|\left({ }^{i j} o_{\mathbf{X Y}}^{\mathbf{I J}}, \mathrm{t}-\mathrm{n}\right)\right\rangle .
$$




\subsection{Collision interpretation}

Intuitively, two systems, $\mathbf{X}$ and $\mathbf{Y}$, interact (collide) over time $\mathrm{t}-1 \ldots \mathrm{t}-\mathrm{n}$. The interaction stops at $\mathrm{t}-$ 1 and the systems are isolated at $t$. This is illustrated below.

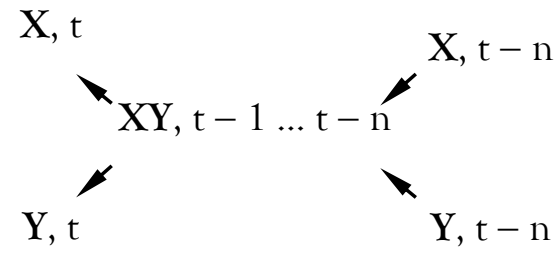

The probabilities of interest are the probabilities that the two isolated systems are in the states ${ }^{k} 0_{\mathbf{X}}^{\mathbf{I}}$, ${ }^{l} o_{\mathbf{Y}}^{\mathbf{J}}$ immediately after the interaction, given that the compound system was in the state ${ }^{i j}{ }_{\mathbf{X Y}}{ }^{\mathbf{I J}}$ at the beginning of the interaction. $\mathbf{n}$ is arbitrary. It is just for illustration.

A somewhat more complete picture is provided in common example. There is a sequence of three interactions: I, II and III. The interactions are linked by sharing systems in the sense that some systems appear in both interactions. II is usually the focus of interest.

$\begin{array}{lll} & \mathrm{X}, \mathrm{t}-\mathrm{n} & \text { Termal molecule } \\ \mathrm{Y}, \mathrm{t}-\mathrm{n} 1 & \mathrm{H} \text { atom } \\ \mathrm{I} & \\ \mathrm{X}, \mathrm{t} & \text { Slower thermal molecule } \\ \mathrm{Y}, \mathrm{t}-\mathrm{n} & \mathrm{H} \text { atom in excited state } \\ \mathrm{X}, \mathrm{t}-\mathrm{n} & \mathrm{H} \text { atom in excited state } \\ \mathrm{Y}, \mathrm{t}-\mathrm{n} & \text { Photon (electromagnetic field) in ground state } \\ \text { II } & & \\ \mathrm{X}, \mathrm{t} & \mathrm{H} \text { atom in ground state } \\ \mathrm{Y}, \mathrm{t}-\mathrm{n} & \text { Photon in excited state } \\ & \mathrm{X}, \mathrm{t}-\mathrm{n} & \text { Photon in excited state } \\ \mathrm{Y}, \mathrm{t}-\mathrm{n} & \text { Detector in ground state } \\ \text { III } & \\ \mathrm{X}, \mathrm{t} & \text { Photon in ground state } \\ \mathrm{Y}, \mathrm{t}-\mathrm{n} & \text { Detector in excited state }\end{array}$

The system that gets passed on is always $\mathbf{X}$ (the object system). The other system (the apparatus system) is always $\mathbf{Y}$. Thus, the same system (e.g. the photon) may be $\mathbf{X}$ in one collision and $\mathbf{Y}$ in another.

One could refer to the same system with the same notation in all interactions. But this would obscure the most important features of the sequence.

This standard story is made somewhat obsolete by quantum electrodynamics. But it suffices for our purposes. We aim only to reconstruct the obsolete theory.

\subsection{Observation interpretation}

Each member of partition $\mathrm{j}$ of (apparatus) system $\mathrm{Y}$ may be viewed as observing (measuring) an observable i of (object) system X.

$$
\mathbf{p}\left({ }^{i} o_{\mathbf{X}}^{\mathbf{i}}, t\right) \mid\left({ }^{i j} o_{\mathbf{X Y}}^{\mathbf{I J}}, \mathrm{t}-1\right)
$$

is the probability that the result of the observation of $\mathrm{j}$ is ${ }^{i} o_{\mathbf{X}}^{\mathbf{i}}$. Together, members of $\mathrm{j}$ may be viewed as a "family" of related observations. 
In the Stern-Gerlach example, $\mathrm{j}$ is a magnetic field of specific orientation. $\mathrm{j}$ is the set of possible orientations of the field. In discussions of this example the state of the apparatus system $\mathbf{Y}$ after the interaction is rarely considered. But, despite spatial separation, it is not obvious that the probabilities of states of $\mathrm{X}$ and $\mathrm{Y}$ are independent (Leighton 2000). If not, the final state at $t$ would be a non-product state.

\section{8. ||-Embedding}

QM determines the probabilities

$$
\mathbf{p}\left({ }^{i} o_{\mathbf{X}}^{\mathbf{i}}, t\right) \mid\left({ }^{i j} o_{\mathbf{X Y}}^{\mathbf{I J}}, \mathrm{t}-1\right),
$$

by embedding the lattice of states of ${ }^{i j} o_{\mathbf{X Y}}^{\mathbf{I J}}$ in the lattice of projection operators of an $\# \mathbf{X} \times \# \mathbf{Y}$ dimensional Hilbert space.

Most generally, for system $\mathbf{X}$, this mapping is a lattice isomorphism onto a sub-lattice of $\mathbf{L}^{\mathrm{P}} \mathbb{H}$,

$$
\begin{gathered}
|| \in\left|\mathbf{L}_{\mathbf{X}}^{\Omega} \triangleright \mathbf{L}^{\mathbf{P H}}\right| \quad o_{\mathbf{X}} \in\left|\mathbf{L}_{\mathbf{X}}^{\Omega}\right| \\
\left|\hat{v}_{\mathbf{X}}^{\mathrm{i}}\right|:=\left\{\left.\right|^{i} \widehat{o}_{\mathbf{X}}^{\mathbf{i}}|| \hat{o}_{\mathbf{X}}^{\mathbf{i}} \in \hat{v}_{\mathbf{X}}^{\mathrm{i}}\right\}
\end{gathered}
$$

is a set of mutually orthogonal elementary projection operators, atoms of $\mathrm{L}^{\mathrm{P}} \mathbb{H}$. These correspond one-one to one-dimensional subspaces of $\mathbb{H}_{X}, \mathrm{U}_{\mid}{ }^{i} \hat{o}_{\mathbf{X}} \mathbf{i} \mid$.

We denote generic normalized vectors in these sub-spaces by

$$
\left|{ }^{i} o_{\mathbf{X}}^{\mathbf{i}}\right\rangle \in \mathrm{U}_{\mid}{ }^{i}{ }_{\hat{o} \mathbf{X}}^{\mathbf{i}} \mid
$$

In the case of interest, it is

$$
\begin{gathered}
|| \in\left|\mathbf{L}_{\mathbf{X Y}}^{\boldsymbol{\Omega}} \triangleright \mathbf{L}^{\mathbf{P H I}}\right| \quad \mathrm{oxY}_{\mathbf{X Y}} \in\left|\mathbf{L}_{\mathbf{X Y}}^{\boldsymbol{\Omega}}\right| \\
\left|{ }^{\mathrm{i}} \hat{v}_{\mathbf{X Y}}^{\mathrm{ij}}\right|:=\left\{\left.\right|^{i j} \widehat{o}_{\mathbf{X Y}}^{\mathbf{i j}}||{ }^{i j} \widehat{o}_{\mathbf{X Y}}^{\mathbf{i j}} \in{ }^{\mathrm{ij}} \hat{v}_{\mathbf{X Y}}^{\mathrm{ij}}\right\}
\end{gathered}
$$

The case of interest here differs from the general case only in that it provides for the some- what idiosyncratic conception of quantum mechanics considered here. The general case is the fundamental idea.

Each embedding determines different probabilities. Any probability function can be obtained from some embedding. Roughly, embeddings "correspond to", or simply "are" probability functions.

\subsection{QM Probabilities}

Conditional probabilities for maximal observables are given by the square of inner product

$$
\left.\mathbf{p}\left({ }^{k} \widehat{o}_{\mathbf{X Y}}^{\mathbf{i j}}, t\right)\left|\left({ }^{i j} \widehat{o}_{\mathbf{X Y}}^{\mathbf{I J}}, \mathrm{t}-1\right)=\right|\left\langle{ }^{k} l_{\mathbf{o}}^{\mathbf{i j}}{ }_{\mathbf{X Y}} \mid{ }^{i j} \widehat{o}_{\mathbf{X Y}}^{\mathbf{I J}}\right\rangle\right|^{2}
$$

This is what essentially defines $\mathbf{M}$, the set of models, in the structuralist jargon.

\subsection{Global QM}

Some features of quantum mechanics cannot be treated within the framework of (1.1). These include creation and annihilation of systems, bound states and atomic spectra. These phenomena are frequently associated with quantum mechanical scattering (Taylor 2006, Ch. 16).

We treat these matters by considering a framework in which systems which exist (are pre- sent), as well as their states, change over time. Within this framework, creation and annihilation of systems is obvious. Two systems become "bound" when they are concatenated to form a com- 
pound system. Photons are systems whose binding and unbinding is commonly noted by a spectrometer.

Formally, this is done with the concept of a "global system" (not to be confused with Immanuel Wallerstein's usage). Roughly, a global system is a set of systems together with a state assignment for each.

To describe this in more detail, we mimic the notation for systems (2.1) but consistently use letters near the beginning of the alphabet for global systems. Let

$$
\begin{gathered}
\mathbb{E} \sim \text { finite set of elementary systems } \\
\mathrm{E} \in \mathbb{E} \sim \text { set of states, } \mathrm{E} \in|\mathrm{SET}| \\
\mathrm{V}_{\mathbb{E}}=\left\langle\left|\mathrm{V}_{\mathbb{E}}\right|, \leq_{\mathrm{E}}^{\mathrm{V}}, \mathrm{V}_{\mathrm{E}}^{\mathrm{V}}, \wedge_{\mathrm{E}}^{\mathbf{V}}, \overline{\mathrm{V}}_{\mathrm{E}}^{\mathbf{V}}, \bar{\Lambda}_{\mathrm{E}}\right\rangle \\
\mathrm{U}_{\mathbb{E}} \in\left|\mathrm{V}_{\mathbb{E}}\right| \sim \text { system configuration. }
\end{gathered}
$$

Each partition of the set of elementary systems is a system configuration where the members of the partition are taken to be systems in the sense of 2.1. Let

$$
\begin{gathered}
\left|\mathrm{V}_{\mathbb{E}}\right| \sim \text { set of system configurations } \\
\mathrm{O}_{\mathrm{UE}} \in \mathrm{SET}\left(\mathrm{U}_{\mathbb{E}}, \mathbb{E}\right) \sim \text { state assignment to members of } \mathrm{U}_{\text {。 }} \\
\left\langle\mathrm{U}_{\mathbb{E}}, \mathrm{O}_{\mathrm{UE}}\right\rangle \sim \text { elementary state of global system } \mathrm{F} .
\end{gathered}
$$

The global system $\mathbf{F}$ is identified with its set of elementary states

$$
\mathrm{F}=\left\{\left\langle\bigcup_{\mathbb{E F}}, \mathrm{OUEF} F\left|U_{\mathbb{E F}} \in\right| \mathrm{V}_{\mathbb{E F}}\right|\right\} \sim \text { set of elementary states of global system } \mathrm{F} .
$$

Members of $\mathrm{F}$ are roughly analogous to "channels" in discussions of quantum mechanical scattering. In contrast to the usual discussions of scattering, all elementary systems appear in all channels, albeit combined in different ways.

Concatenation for global systems is

$$
\begin{aligned}
& \mathrm{FG} \subset \mathrm{F} \times \mathrm{G} \sim \text { concatenation for global systems } \\
& \left\langle\left\langle U_{\mathbb{E F}}, \mathrm{OUEFF}_{\mathbb{F}}\right\rangle,\left\langle\mathrm{U}_{\mathbb{E G}}, \mathrm{O}_{\mathbb{E G}}\right\rangle\right\rangle \in \mathrm{F} \times \mathrm{G} .
\end{aligned}
$$

We consider a finite set of set of global systems analogous to $\mathrm{S}$ (2.1). Let

$$
\begin{aligned}
& \mathrm{E} \sim \text { finite set of sets of elementary states, set of global systems, analogous to } \mathrm{S} \text {. } \\
& \mathfrak{E}:=\bigcup_{\mathbf{F} \in \mathbf{E}} \text { Pot } \mathbf{F} \sim \text { set of all states of all global systems. } \\
& \mathfrak{E} \times \mathrm{T} \sim \text { set of all } \mathrm{T} \text {-sequences of members of } \mathfrak{E} \text {. } \\
& \mathbf{B}_{\mathfrak{E} \times \mathbf{T}} \sim \text { Boolean algebra of subsets of } \mathbf{T} \text {-sequences of members of } \mathfrak{E} \text {. }
\end{aligned}
$$

Partitions of $\mathbf{F}$ and their members are indexed so that $o_{\mathbf{F}}^{\mathbf{m}} \in\left|\mathbf{V}_{\mathbf{F}}\right|$ is an indexed partition of $\mathbf{F}$, and ${ }^{m} O_{\mathbf{F}}^{\mathbf{m}} \in O_{\mathbf{F}}^{\mathbf{m}}$ is an indexed global state in partition $o_{\mathbf{X}}^{\mathbf{i}}$.

As above, the set of observables for global system $\mathrm{F}$ is

$$
\Omega_{\mathrm{F}} \subseteq\left|\mathrm{V}_{\mathrm{F}}\right| \text {. }
$$

Again, we do not explain how the member of the partition containing the state of the global system can be determined. Roughly, it is done by each of the members of $\mathbf{F}$ interacting with some other system in the manner described in (2.5-2.6) above.

We require

1) $\Omega_{\mathrm{F}} \neq \Lambda$

2) $\mathrm{U}_{\mathrm{F}} \in \Omega_{\mathrm{F}} \Rightarrow \uparrow_{\mathrm{U}_{\mathrm{F}}} \subset \Omega_{\mathrm{F}}$

3) $U_{\mathrm{F}} \in \Omega_{\mathrm{F}} \Rightarrow U_{\mathrm{F}} \times \mathrm{G} \in \Omega_{\mathrm{FG}}$. 
The partition ordering, restricted to $\Omega_{\mathrm{F}}$, is a sub-lattice of $\mathrm{V}_{\mathrm{F}}$,

$$
\mathrm{L}_{\mathrm{F}}^{\Omega} \check{L A T}_{\mathrm{F}} \mathrm{V}_{\mathrm{F}}
$$

Atoms of $\mathrm{L}_{\mathrm{F}}^{\Omega}$ are $\hat{v}_{\mathbf{F}}^{\mathbf{m}}$.

Global QM is concerned with certain conditional probabilities derived from probability functions

$$
\mathrm{p} \in \operatorname{PROB}\left(\mathrm{B}_{\mathfrak{E} \times \mathrm{T}}\right)
$$

Most generally, we consider, for $0 \in \mathfrak{E}$.

$$
\mathrm{p}(\mathrm{o}, \mathrm{t}):=\mathrm{p}(\langle\mathrm{o}, \mathrm{t}\rangle)
$$

Note that

$$
{ }^{m} O_{\mathbf{F}}^{\mathbf{m}} \in \mathfrak{E}
$$

so that

$$
\mathbf{p}\left({ }^{m} O_{\mathbf{F}}^{\mathbf{m}}, t\right)
$$

is defined.

Global QM is concerned with conditional probabilities of the form

$$
\mathrm{p}\left({ }^{\mathrm{m}} O_{\mathbf{F}}^{\mathbf{m}}, \mathrm{t}\right) \mid\left({ }^{\mathrm{mn}} \mathrm{O}_{\mathbf{F G}}^{\mathbf{m n}}, \mathrm{t}-\mathrm{n}\right) .
$$

Global QM determines these probabilities by embedding the lattice of states of ${ }^{\mathrm{mn}} \boldsymbol{O}_{\mathbf{F G}}^{\mathbf{m n}}$ in the lattice of projection operators of an \#F $\mathrm{x} \# \mathrm{G}$-dimensional Hilbert space.

$$
\left|\mathbf{L}_{\mathbf{F G}}^{\mathbf{\Omega}} \triangleright \mathbf{L}^{\mathbf{P H}}\right| \quad \text { OFG }_{\mathrm{FG}} \in \mathbf{L}_{\mathbf{F G}}^{\mathbf{\Omega}} \mid,
$$

so that

$$
\left.\mathbf{p}^{(o \circ} \widehat{o}_{\mathbf{F G}}^{\mathbf{m n j}}, t\right)\left.\left|\left({ }^{m n} \widehat{o}_{\mathbf{F G}}^{\mathbf{m n}}, \mathrm{t}-1\right)=\right|\left\langle\left.{ }^{o n p} \widehat{o}_{\mathbf{F G}}^{\mathbf{m n}}\right|^{m n} \widehat{O}_{\mathbf{F G}}^{\mathbf{m n}}\right\rangle\right|^{2} .
$$

Note that systems are a special case of global systems in which system configurations are singletons, i.e.

$$
S=\left\{F\left|U_{\mathbb{E F}} \in\right| \mathrm{V}_{\mathbb{E F}} \mid \Rightarrow \# \mathrm{U}_{\mathbb{E F}}=1\right\} .
$$

Thus, QM is a special case of global QM. A more austere exposition of these ideas would first describe global QM and then the special case of QM. While elegant, this does not illuminate the essential ideas.

The Pauli Exclusion Principle may be viewed as part of the apparatus of particle mechanics. It plays a role roughly analogous to that of conservation laws in restricting possible particle paths.

For 2-component systems, the Pauli Exclusion Principle requires that

$$
\left|\mathrm{i} \hat{o} \hat{o}_{\left[\mathbf{X}_{1}, \mathbf{X}_{2}\right]}^{\mathbf{i}}\right\rangle=\left|\mathrm{i} \hat{o}_{\mathbf{X}_{1}}^{\mathbf{i}}\right\rangle\left|\mathrm{i} \widehat{o}_{\mathbf{X}_{2}}^{\mathbf{i}}\right\rangle \pm\left|\mathrm{i} \hat{o}_{\mathbf{X}_{2}}^{\mathbf{i}}\right\rangle\left|\mathrm{i} \widehat{o}_{\mathbf{X}_{1}}^{\mathbf{i}}\right\rangle
$$

with the '-' used for particles with $\operatorname{spin}=1 / 2$ (Fermions) and the ' + ' for particles with spin $=1$ (Bosons).

$$
\mathbf{X}_{1}=\mathbf{X}_{2} \Rightarrow\left|\mathrm{i}_{\left[\mathbf{X}_{1}, \mathbf{X}_{2}\right]}^{\mathbf{i}}\right\rangle=0
$$

i.e. the state vector is 0 and thus probabilities are 0 for identical spin $=1 / 2$ (Fermions). Intuitively, identical spin $=1 / 2$ (Fermions) cannot be in the same state.

For arbitrary integer w, all pairwise exchanges of particle order are considered with '-' and '+'. 


\section{Examples}

This general picture will be filled out with details of specific examples. To carry this out we depart slightly from the temporal history of discovery described in Feynman, Leighton and Sands (1965, Sec. 1). We consider each particle type and the particle tracks associated with it. As indicated above (1), some care has been taken to clearly distinguish cloud/bubble chamber trajectories from tracks and particle tracks.

\subsection{Atoms and molecules}

Atoms and molecules are a particle type with values charge and mass. More precisely,

$$
\text { atom } \vee \text { molecule }=\left\{\left\langle\text { mass, } \mathbb{R}^{(+}\right\rangle \text {, charge }\{+, \Lambda,-\}\right\}
$$

where ' $\Lambda$ ' denotes no charge value. Thus, an atom or molecule has a property named 'mass' whose possible values are strictly positive real numbers and a property named 'charge' whose values are the set $\{+, \Lambda,-\}$.

We assume a theory of chemistry, the theory of atoms and how they combine to form molecules. This theory also tells us the size (spatial dimensions of atoms).

Roughly, A mole of compound contains Avogadro's number of atoms, so each atom must weigh atomic weight/ Avagadro's \#.

This is essentially 19th century chemistry, Lavoisier, Dalton.

Tracks of atoms and molecules are visible only when they converge or diverge. At a diverging point an invisible atom track disappears and multiple tracks begin. These are tracks of a positively charged nucleus and a negatively charged electron (see 4.2). At a converging point multiple tracks disappear and an invisible atom or molecule track begins.

\subsection{Electrons}

Electrons are a particle type with values mass, charge, and spin. More precisely,

$$
\text { electron }=\left\{\left\langle\mathrm{m}, \mathbb{R}^{(+}\right\rangle, \mathrm{q}\left\{\mathrm{n} 1 \mid \mathrm{n} \in \mathbb{I}^{(+}\right\}, \operatorname{spin}\left\{\mathrm{n} / 2 \mid \mathrm{n} \in \mathbb{I}^{(+}\right\}\right\} .
$$

These properties are seen in particle tracks in a variety of contexts. Among these contexts are cathode rays.

Electrons are elementary particles (2.2).

Cathode rays are electron tracks. They are produced by heating a metal wire (cathode) enclosed in a cylindrical glass tube from which most of the air has been evacuated. A second wire (anode) is in the tube and an electric field exits between the cathode and anode. The tube extends some distance beyond the anode and the track passes through a hole in the anode. The residual gas in the tube glows along the axis of the tube producing a track extending some distance beyond the anode. The detector for this track is the tube, or more precisely, the residual gas in the tube.

A magnetic field in the direction perpendicular to the axis of the tube produces a track which is a segment of a circle of radius $r$.

A particle is assigned to this track. Assuming the particle obeys the laws of classical electromagnetic theory this determines $\mathrm{q} / \mathrm{m}$, the charge/mass ratio, for the particle.

A second experiment (Millikan oil drop) varying the strength of electric field reveals that the mass of the particle is always a small integral multiple of a basic mass, $\mu^{-}$.

At the risk of some terminological confusion, we call a particle with this basic mass and negative charge 'an electron'. We do this simply to connect with more traditional terminology. An electron is not the particle type electron.

Alternatively, we observe particle tracks. Various electro-magnetic field strengths perpendicular to the axis of the cathode ray tube produce curved paths of varying radii. 
We invent a particle type

$$
\text { electron }=\left\{\left\langle\mathrm{m},\left\{\boldsymbol{\mu}^{-}\right\}\right\rangle, \mathrm{q}\{-1\}\right\}=\left\{\left\langle\text { mass, }\left\{\boldsymbol{\mu}^{-}\right\}\right\rangle \text {, charge }\{-1\}\right\}
$$

to account for this. The spin property is not evident in this context. Below we will treat other elementary particles, quarks and leptons in this way.

\subsection{Protons}

Compton scattering of electrons, analyzed by classical mechanics, indicates that electrons surround a positively charged "core”. The core of the hydrogen atom is called a 'proton'. Free protons are not common. But it is possible to produce proton beams (Messiah 1961) and to observe proton tracks (Birkhoff 1967).

At this time proton properties are the subject of active investigation. See

$$
\begin{gathered}
\text { https://en.wikipedia.org/wiki/Proton_spin_crisis } \\
\text { https://en.wikipedia.org/wiki/Proton_decay }
\end{gathered}
$$

and literature cited therein.

Roughly, protons are a particle type with values mass, charge, and spin. More precisely,

$$
\begin{array}{r}
\text { proton }=\left\{\left\langle\mathrm{m}, \mathbb{R}^{(+}\right\rangle, \mathrm{q}\left\{\mathbb{R}^{(+} \mid \mathrm{n} \in \mathbb{I}^{+}\right\} \text {, spin }\left\{\mathrm{n} / 2 \mid \mathrm{n} \in \mathbb{I}^{(+}\right\}\right\} . \\
\quad=\left\{\left\langle\text { mass, } \mathbb{R}^{(+}\right\rangle \text {, mass }\{\sim 1\}, \mathrm{q}\{1\}, \text { spin }\left\{\mathrm{n} / 2 \mid \mathrm{n} \in \mathbb{I}^{+}\right\}\right\} .
\end{array}
$$

Protons are not elementary. Roughly, they are composed of 2 up quarks, 1 down quark. They are classified as fermions in the classification scheme described in 3.10 .

\subsection{Neutrons}

Ionized He particles have the mass $2 \mathrm{X}$ the mass of ionized $\mathrm{H}$ (protons) but no charge. This is shown by their particle tracks in an electromagnetic field. Ionized particles with $\mathrm{nX}$ the mass of ionized $\mathrm{H}$ but no charges are also observed.

Rutherford and others noted the disparity between the atomic number of an atom, or number of positive charges, and its mass computed in atomic mass units. The atomic number of an atom is usually about half its atomic mass. In 1920 Rutherford suggested that the disparity could be explained by the existence of an uncharged particle with mass identical to that of the proton within the atomic nucleus.

This is actually a bit more complicated. See references in

$$
\text { https://en.wikipedia.org/wiki/Discovery_of_the_neutron }
$$

Neutrons in an electro-magnetic field do not leave a particle track. However, a particle track can be inferred from particle tracks produced by "secondary ionization" terminology mine (Altraus \& Sard 1953). It is questionable whether this fact justifies taking particle tracks as evidence for neutrons. Here we assume that these inferred particle tracks are evidence for neutrons.

Neutrons are classified as baryons in the classification scheme described in 3.10.

\subsection{Photons}

Photons (and other mediating particles) leave tracks consisting of tracks of mediated particles. Photons leave tracks consisting of positive ion tracks and electron tracks. These are observed as cloud/bubble chamber photographs in the manner described in Gillespie (1994).

In the traditional account, photons are emitted when electrons change to lower energy value; absorbed when they change to a higher. These respectively produce emission and absorption spectral lines. Conditional probabilities derived from $\mathbf{p}$ are interpreted as the intensities of these spectral lines. 
Two things are calculated to determine the particle tracks of mediating particles: A) the probabilities of velocities in all directions (scattering cross sections $(\sigma))$; B) the probabilities of all times spent in these directions (decay rates $(\Gamma)$ ) (Gleyzes et al. 2007, p.197).

\subsection{Mesons}

Since particles are assumed to satisfy the laws of particle mechanics and electrodynamics 2.1, the protons in nucleus should repel each other and move apart. A "force" holding them together is assumed. This is called the 'strong force'. It is mediated by mesons. The mass of mesons can be calculated. This permits tracks produced by cosmic rays to be identified as meson tracks. There are two mesons of ( $\pi$ and $\mu$, sometimes called Yukawa mesons after their discoverer).

I have not been able to find a report of Yukawa's mass calculation. A reconstruction appears here

$$
\text { http://math.ubooks.pub/Books/ON/M1/1704/C33S2M004.html }
$$

A description of the circumstances of the publication appears here

$$
\text { http://www.f.waseda.jp/sidoli/Brown_1986.pdf }
$$

\subsection{Antiparticles}

Theoretical considerations about conservation of relativistic momentum-energy led Dirac and others to predict the existence of antiparticles. Every particle has an antiparticle with the same mass and opposite charge. Some particles are their own antiparticles.

Antiparticles produce tracks in the same ways that particles do. These can be observed in essentially the same way.

These tracks could have been observed before the theoretical considerations predicted them. It is just an 'historical accident' that they were not.

Crossing symmetry describes ways that particle tracks may branch, provided the branching also satisfies energy conservation.

\subsection{Neutrinos}

In 1930, experiments seemed to suggest that some nuclear decays did not conserve energy - less energy was detected in the final state than in the initial one. Wolfgang Pauli proposed that the energy was being carried off by an unseen particle, one that had no electric charge and interacted only via the weak interaction, in which case it would pass right through normal detectors without any effect. Yet it would carry off energy. If that were true, certain predictions could be made for the motion of the other particles that emerged in the decay, and those were verified, so within a few years most experts were convinced from the indirect evidence that "neutrinos" indeed existed. It wasn't until 1958, almost thirty years after they were postulated, that they were directly detected. Neutrinos are often denoted by the Greek letter nu (v) (Kane 2016).

\section{Compare to}

There are three charged leptons: the electron and its two heavier siblings, the muon and the tauon. The muon was discovered in cosmic ray experiments and was initially mistaken for the pion, the particle predicted by Yukawa. Its mass is $106 \mathrm{MeV} / \mathrm{c} 2$, about two hundred times the mass of the electron. The tauon, which was discovered in 1975 in electron-positron annihilation experiments, is much heavier, about $1777 \mathrm{MeV} / \mathrm{c} 2$. The tauon's properties have been much less studied than those of the two lighter charged leptons but all appear to be structureless elementary spin-particles (Martin 2011).

In Martin (2011), there is no mention of theoretical considerations that predict them. This is a somewhat misleading omission. It misled me for a time.

The two are not really incompatible but Martin might be misleading. 
Recall (2.) that all particles are subject to some force, though not necessarily a familiar one. This is why we assume the existence of a "weak force" mediated by a W boson.

\subsection{Lepton/Baryon number}

Lepton numbers and baryon numbers assigned to particle types make it possible to formulate conservation laws "conservation of lepton number "conservation of baryon number", which further restricts the set of particle tracks. Lepton number is conserved in a strong interaction, but not in a weak interaction. Baryon number is conserved in any interaction.

\subsection{Strange particles}

Strange particles provide paradigm examples of the view taken here. Particle tracks are observed first; then particle types are devised to reproduce these tracks.

[...] Rodchester and Butler [17] published the cloud chamber photograph show in Figure 1.8 Cosmic ray particles enter from the upper left and strike a lead plate, producing a neutral particle whose presence is revealed when it decays into two charged secondaries, forming an upside- down ' $\mathrm{V}$ ' in the lower right. [...] another neutral ' $\mathrm{V}$ ' particle was found by Anderson's group [...] but this time the products were a $\mathrm{p}^{+}$ and a $\pi^{-}$.

Conservation of baryon number explains why the decay

$$
\mathrm{p}^{+} \rightarrow \mathrm{e}^{+}+\gamma
$$

is not observed (Griffiths 2000, p. 31).

\subsection{Eightfold way}

This is essentially a classification scheme for particle types. New particle types (additions to the particle zoo) are predicted in the sense that there are gaps in the scheme. Particle types that fill these gaps are predicted.

\subsection{Quarks}

Quarks are a particle type which serves to explain the particle types predicted by the eightfold way (4.10)

\subsection{Gluons}

Gluons are a mediating particle type. They play a role for the strong force analogous to that of the photon for the electromagnetic force.

\section{References}

Altraus, E. J. and R. D. Sard (1953), "A Cloud-Chamber Study of Neutron Production by Sea-Level Cosmic Rays with Particular Reference to $\mu$-Mesons Stopped in Lead", Physical Review 91(2): 373-384.

Ballentine, L. E. (1986), "Probability Theory in Quantum Mechanics”, American Journal of Physics 54(10): 883-889.

Ballentine, L. E., (1998), Quantum Mechanics: A Modern Development, Singapore: World Scientific Publishing Co.

Balzer, W., Moulines, C. U. and J. D. Sneed, (1987), An Architectonic for Science: The Structuralist Program, Dordrecht: Reidel.

Bartelborth, T. (2000), "An Axiomatization of Classical Electrodynamics", in Balzer, W., Sneed, J. D and C. U. Moulines (eds.), Structuralist Knowledge Representation: Paradigmatic Examples, Amsterdam: Rodopi, pp. 333-352.

Birkhoff, G. (1967), Lattice Theory, Providence, RI: American Mathematical Society. 
Caves, C. M. (n.d.), Multiple Systems, the Tensor-Product Space and the Partial Trace. http://info.phys.unm.edu/ ${ }^{\sim}$ caves/courses/qinfo-f05/lectures/tensorproduct.pdf.

Caves, C. M., Fuchs, C. A. and S. Rüdiger (2002), "Quantum Probabilities as Bayesian Probabilities”, Physical Review A 65: 022305.

Cohen-Tannoudji, C., Dupont-Roc, J. and G. Grynberg (1989), Photons and Atoms: Introduction to Quantum Electrodynamics, New York: John Wiley \& Sons.

Condon, E. U. and G. Shortley (1951), The Theory of Atomic Spectra, Cambridge: Cambridge University Press.

Dirac, P. A. M. (1958), The Principles of Quantum Mechanics, Oxford: Clarendon Press.

Fano, U. and L. Fano (1959), Basic Physics of Atoms and Molecules, New York: Wiley.

Feynman, R. P. (1951), “The Concept of Probability in Quantum Mechanics”, in Neyman, J. (ed.), Proceedings of the Second Berkeley Symposium on Mathematical Statistics and Probability, Berkeley: University of California, pp. 533-541.

Feynman, R. P., Leighton, R. B. and M. Sands (1965), The Feynman Lectures on Physics, Vol. III: Quantum Mechanics, Reading, MA: Addison-Wesley.

Gillespie, D. T. (1994), "Why Quantum Mechanics Cannot be Formulated as a Markov Process", Physical Review A 49: 1608.

Gleyzes, S., Kuhr, S., Guerlin, C., Bernu, J., Deléglise, S., Busk Hoff, U., Brune, M., Raimond, J. M. and S. Haroche (2007), "Quantum Jumps of Light Recording the Birth and Death of a Photon in a Cavity", Nature 446(7133): 297-300.

Griffiths, D. T. (2000), Introduction to Elementary Particles, Weinheim: Wiley-VCH.

Halmos, P. R. (1958), Finite-Dimensional Vector Spaces, Princeton, NJ: Van Nostrand.

Held, C. (2008), “The Kochen-Specker Theorem”, The Stanford Encyclopedia of Philosophy (Winter 2008 Edition), Zalta, E. N. (ed.), <http://plato.stanford.edu/archives/win2008/entries/kochen-specker/>.

Herbut, F. (1992), "Quantum Interference Viewed in the Framework of Probability Theory”, American Journal of Physics 60(2): 146-150.

Hooker, C. A. (1975), The Logico-Algebraic Approach to Quantum Mechanics, Vol. I, Dordrecht: Reidel.

Jammer, M. (1966), The Conceptual Development of Quantum Mechanics, New York: McGraw-Hill.

Jaynes, E. T. (2003), Probability Theory: The Language of Science, New York: Cambridge University Press.

Joos, G. (1958), Theoretical Physics, 3rd ed., New York: Hafner Publishing Company.

Kane, G. (1958), Modern Elementary Particle Physics, New York: Cambridge University Press, 2nd ed.

Kane, G. (2016), Supersymmetry and Beyond: From the Higgs Boson to the New Physics, Cambridge, MA: Basic Books.

Koopman, B. O. (1995), "Quantum Theory and the Foundations of Probability", in MacColl, L.A. (ed.), Applied Probability, New York: McGraw-Hill, pp. 97-102.

Krantz, D. H., Luce, D., Suppes, P. and A. Tversky (1971), Foundations of Measurement, Vol. 1, New York: Academic Press.

Kuhn, T. S. (1978), Black-Body Theory and the Quantum Discontinuity, Chicago: University of Chicago Press.

Martin, B. R. (2011), Particle Physics, Oxford, UK: Oneworld Publications.

Moyal, J. E. (1949), "Quantum Mechanics as a Statistical Theory”, Proceedings of the Cambridge Philosophical Society 45: $97-124$.

Messiah, A. (1961), Quantum Mechanics, Vols. I and II, Amsterdam: North-Holland.

Nielson, M. A. and I. L. Chuang (2000), Quantum Computation and Quantum Information, Cambridge: Cambridge University Press

Peres, A. (1995), Quantum Theory: Concepts and Methods, Dordrecht: Kluwer.

Preskill, J. (2004), Lecture Notes, Pasadena, CA: California Institute of Technology, http://www.theory.caltech.edu/people/preskill/ph229/\#lecture 2004. 
Schmidt, H. J. (2003), "Structuralism in Physics”, The Stanford Encyclopedia of Philosophy (Spring 2003 Edition), Zalta, E. N. (ed.), http://plato.stanford.edu/archives/spr2003/entries/physics-structuralism.

Scott, G. W. (1939), "Focused Beam Source of Hydrogen and Helium Ions”, Physical Review 55 (1939): 954-959.

Sneed, J. D. (1964), The Projection Postulate and Quantum Mechanical Measurement, unpublished Ph.D. dissertation, Stanford: Stanford University.

Sneed, J. D. (1970), "Quantum Mechanics and Classical Probability Theory”, Synthese 21: 34-64.

Sneed, J. D. (1979), The Logical Structure of Mathematical Physics, Dordrecht: Reidel, 2nd ed.

Sneed, J. D. (2011), "Prolegomena to a Structuralist Reconstruction of Quantum Mechanics”, Metatheoria 1(2): 93. 130.

Stegmüller, W. (1979), The Structuralist View of Theories, New York: Springer.

Stevens, S. S. (1946), "On the Theory of Scales of Measurement”, Science 103: 677-680.

Taylor, J. R. (2006), Scattering Theory: The Quantum Theory of Nonrelativistic Collisions, Mineola, NY: Dover.

von Neumann, J. (1955), Mathematical Foundations of Quantum Mechanics (translated from the German edition by R. T. Beyer), Princeton: Princeton University Press.

Wigner, E. P. (1932), “On the Quantum Correction for Thermodynamic Equilibrium”, Physical Review 40: 749-759.

Wilczek, F. (1990), Fractional Statistics and Anyon Superconductivity, Singapore: World Scientific Publishing Co.

Zurek, W. H. (2003), "Decoherence, Einselection and the Quantum Origins of the Classical”, Review of Modern Physics 70(3): 715-775. 